\title{
A Major Multifactorial Urinary Retention in an Elderly Woman: A Case Report
}

\author{
De Luca $E^{*}$, Inelmen EM, Pintore G, Catanzaro S and Manzato E \\ Department of Medicine-DIMED, Geriatrics Section, University of Padua, Italy
}

\begin{abstract}
This case report concerns an 89-year-old woman who tolerated a urinary retention of $5000 \mathrm{ml}$ due to longstanding asymptomatic bladder atony. It had a multifactorial origin because the patient had a lengthy history of benzodiazepine abuse for severe reactive depression. Furthermore, a large drugs assumption, cognitive impairment and low mobilization may have influenced the severity of her condition. She had never worried about her distended abdomen because her micturition was good cause of overflow incontinence.
\end{abstract}

To our knowledge, this is the first report of an impaired elderly patient developing major urinary retention also as a result of using benzodiazepines that may have acquired a fundamental rule as trigger factor.

Keywords: Urinary retention; Elderly; Benzodiazepine; Adverse drug reactions

\section{Background}

Lower urinary tract disorders are a typical finding in the elderly because of the physiological changes that occur with aging. Comorbidities and acute diseases adversely affecting the urinary tract also often coincide with old age [1].

Urinary retention is a common finding and can be described as the inability to empty the bladder completely after detrusor contraction and voluntary micturition [1]. It is generally seen in elderly men because the male gender's genito-urinary apparatus is particularly susceptible to physiological and pathological changes. This situation is exacerbated by functional decline and immobility, and often correlates with bladder outlet obstruction as a consequence of benign prostate enlargement [2].

Urinary retention is uncommon in women and tends to be asymptomatic. It is more likely in elderly women with mobility problems and comorbidities. Being unusual, this condition in women has received little attention in epidemiological research and clinical reports, but one such study indicated that the incidence of acute urinary retention in women is 7 per 100,000 populations a year [3]

It is also important to distinguish between acute and chronic bladder distension, because the former is usually painful and patients are referred to physicians as emergency cases, while chronic retention is often painless and patients are unaware of the problem. If it is not investigated, chronic urinary retention may lead to overflow incontinence and upper urinary tract dysfunction [4].

\section{Factors affecting micturition in elderly patients}

Given the complexity of the mechanism behind micturition, it is easy to understand how chronic disorders (impaired detrusor contraction with coordination difficulties on voiding, anatomical changes, or neurological problems [1], as occur in diabetic patients [4] or acute conditions such as infections can interfere with this function. Another important, but often underestimated factor that can interfere with the mechanism of micturition in elderly patients is the use of medications [5].

Many drugs may have more severe negative effects on micturition in the elderly because they often take more than one type of medication, and this exposes them to a higher risk of developing drug-related urinary retention. Due to their concomitant use of several drugs, physicians find it difficult to separate drug reactions from clinical problems. The most important issue to consider in assessing adverse drug reactions is whether there is any link between a drug's intake and a clinical event. Adverse drug reactions may be identified with the aid of the Adverse Drug Reaction (ADR) probability scale [6], a tool that points to a definite (>9), probable (5-8), possible (1-4), or doubtful $(<0)$ drug reaction based on a patient's answers to 10 questions. In reports in the literature, urinary retention has been correlated with the use of anticholinergic medication that causes dry mouth, sedation and constipation, such as antipsychotic and antidepressant drugs (SSRIs), anticholinergic respiratory agents, opioids and anesthetics, benzodiazepines, NSAIDs, a-adrenoreceptors, detrusor relaxants, and calcium channel antagonists [5]. But drug-related urinary retention has been studied very little, so no data are available on its incidence. Some observational studies have suggested that about $10 \%$ of cases are likely to correlate with the use, or abuse of medication [5].

Given the shortage of publications on the matter, we report here on a case of urinary retention in an elderly woman correlating, as contributory causes, with the use of high doses of benzodiazepine (bromazepam), occasionally associated with lercanidipina and pericyazine, which went unnoticed for some time.

\section{Case Presentation}

An 89-year-old woman, C.A., with hypertension, chronic cerebrovascular disease and major electrolyte imbalance was admitted to hospital.

A comprehensive geriatric assessment revealed severe cognitive impairment, loss of functional integrity and severe anxious-depressive syndrome. She had been abusing bromazepam for some time to combat the effects of her longstanding deflected mood; she also took

*Corresponding author: Elisabetta De Luca, MD, Department of Medicine, DIMED Geriatric Section, University of Padova, Via Giustiniani 2, 35128 Padova, Italy, Tel: 0039-0498218493; Fax: 0039-0498211218; E-mail: elisabetta121283@libero.it

Received August 02, 2012; Accepted August 14, 2012; Published August 16, 2012

Citation: De Luca E, Inelmen EM, Pintore G, Catanzaro S, Manzato E (2012) A Major Multifactorial Urinary Retention in an Elderly Woman: A Case Report. J Clin Case Rep 2:182. doi:10.4172/2165-7920.1000182

Copyright: (C) 2012 De Luca E, et al. This is an open-access article distributed under the terms of the Creative Commons Attribution License, which permits unrestricted use, distribution, and reproduction in any medium, provided the original author and source are credited. 
lercanidipina and occasionally pericyazine as well. Her excessive, uncontrolled use of benzodiazepines caused postural instability and she had a history of accidental falls causing fractures and cranial traumas; she had also fallen a few days before she was hospitalized. She was found constipated, which was probably due mainly to an adverse reaction to the benzodiazepines, but was also associated with her poor functional conditions. Her consequent frequent use of laxatives was responsible for a major electrolyte imbalance detected on admission to hospital.

The patient reported having had no problems with micturition at home, but it emerged from her clinical history that she had experienced bladder stagnation after a previous hospital stay.

Soon after arriving in our ward, the patient urinated with ease. She seemed agitated and revealed compulsive ideas concerning her psychoactive drug use.

Clinical examination revealed a distended abdomen, a condition the patient had been experiencing for some time but had not worried about. She was uncooperative during the clinical examination and percussion of the abdomen initially failed to produce the classical signs of stagnation, probably due to intestinal loops masking the bladder's fullness. We assumed that overflow incontinence had masked the problem, given that the patient had urinated spontaneously on arrival in the ward.

\section{Investigations}

Abdominal X-ray (performed with constipation or a neoplastic mass in mind) revealed a severely enlarged bladder (Figures 1), and catheterization released $5000 \mathrm{ml}$ of urine. The ADR Probability Scale [6] came to our aid: assessing each type of medication the patient was taking at home by answering the 10 questions with the data in our possession, the patient scored 6 for bromazepam, 0 for atenolol, 0 for zofenopril plus hydrochlorothiazide, 4 for lercanidipina, and 4 for pericyazine. A score of 6 indicates that the patient's use of
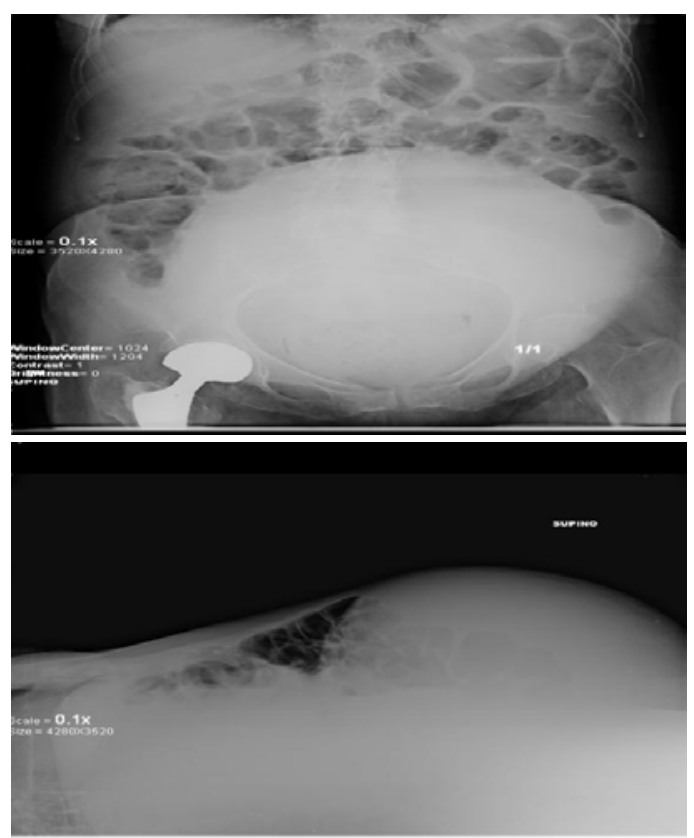

Figure 1: Abdominal radiography showing the bladder in two projections. benzodiazepines was "probably" related to her adverse drug reaction, while a score of 4 indicates a possible relationship with an adverse drug reaction.

\section{Outcome and Follow Up}

We hypothesized that a longstanding use of bromazepam associated with lercanidipine and pericyazine in a cognitively impaired woman with mobility problems and constipation had induced a chronic bladder atony that led to massive urine stagnation.

This impression was confirmed by the fact that urinary stagnation recurred $(4000 \mathrm{ml})$ when an attempt was made to remove the catheter before discharging the patient, even though the likely causes of her urinary retention had been removed.

\section{Discussion}

This report concerns an elderly woman hospitalized for constipation and electrolyte imbalance, cognitively impaired and bedridden, who revealed severe chronic urinary retention related, as contributory causes, to her benzodiazepine abuse and concomitant use of antipsychotic drugs and calcium channel antagonists. There are a few reports in the literature of urinary retention relating generally to the use of antidepressants [7] or diphenhydramine [4], but not to the best of our knowledge to bromazepam. Comorbidities and functional decline are characteristic of elderly people and their consequent use of numerous drugs predisposes them to adverse reactions, which often go unnoticed by physicians and unmentioned by patients. While physicians should aim to avoid polypharmacy as far as possible, it is important to assess the risks and benefits of unavoidable pharmacological treatments, overall if cognitive impairment and poor functional status are present. Particular caution is needed in prescribing benzodiazepines for these elderly patients because of their negative effects, which often go unheeded or misunderstood.

\section{Conclusion}

Considering the difficulty of correlating adverse reactions with the drugs responsible for them, it is always important to obtain a thorough medical history to investigate every aspect of a patient, preferably taking a multidimensional approach. This can highlight the importance of only administering benzodiazepines to the elderly when they are genuinely warranted, given their often masked adverse effects; furthermore, avoiding polypharmacy whenever possible is a good observance, in elderly patients, overall if impaired and with low functional status.

\section{References}

1. Mevcha A, Drake M (2010) Etiology and management of urinary retention in women. Indian J Urol 26: 230-235.

2. Abrams $P$ (1999) Bladder outlet obstruction index, bladder contractility index and bladder voiding efficiency: three simple indices to define bladder voiding function. BJU Int 84: 14-15.

3. Klarskov P, Andersen JT, Asmussen CF, Brenøe J, Jensen SK, et al. (1987) Acute urinary retention in women: a prospective study of 18 consecutive cases. Scand J Urol Nephrol 21: 29-31.

4. Shimizu T, Tokuda Y (2011) Acute urinary retention. Intern Med 50: 22-55

5. Verhamme K, Sturkenboom M (2008) Drug-induced urinary retention incidence, management and prevention. Drug Saf 31: 373-388.

6. Naranjo CA, Busto U, Sellers EM et al (1981) A method for estimating the probability of adverse drug reactions. Clin Pharmacol Ther 30: 239-245.

7. Garakani A (2010) Urinary retention with a high dose of escitalopram. Gen Hosp Psychiatry 32: e11-e12. 